\title{
Anti-inflammation effects of the total saponin fraction from Dioscorea nipponica Makino on rats with gouty arthritis by influencing MAPK signalling pathway
}

\author{
Qi Zhou ${ }^{1 *}$ D, Hui Juan Sun², Shu Min Liu', Xi Hong Jiang², Qiu Yue Wang², Shuang Zhang ${ }^{2}$ and Dong Hua Yu
}

\begin{abstract}
Background: Dioscorea nipponica Makino is widely used in traditional Chinese medicine to treat gouty arthritis.

Methods: Sixty male Wistar rats were divided into six groups: the normal group, model group, colchicine group $(\mathrm{COL})$ and three total saponin groups (RDN) (high dose [160 mg/kg], middle dose [80 mg/kg] and low dose [40 mg/ $\mathrm{kg}]$ ). HE staining was used to detect the histopathologic changes of the synovial tissue of joint.

Immunohistochemical method was used to detect the protein expressions of P-38, p-P38, JNK, p-JNK, ERK1/2, pERK1/2, MEK1/2, p-MEK1/2, MKK4, p-MKK4, ICAM1, VCAM1, and PPARY in the synovial tissue of joint. Realtime PCR and WB methods were used to detect the mRNA and protein expressions of PPARy and AdipoR2 in the synovial tissue of joint. The contents of CXCL1 and ADP in the blood serum were measured by Elisa method.

Results: Our study showed that RDN could improve the situation of the synovial tissue, reduce the protein expressions of MKK4, p-MEK1/2, p-JNK, p-ERK1/2, ICAM1. They could also decrease the content of CXCL1 and increase the content of ADP in the blood serum.

Conclusion: RDN has good effect of anti-inflammation. This is in part realized by influencing MAPK signalling pathway. It provides a new visual angle to reveal the mechanism of RDN to treat GA.
\end{abstract}

Keywords: Dioscorea nipponica Makino, Gouty arthritis (GA), Mitogen-activated protein kinase (MAPK), Peroxisome proliferators-activated receptors (PPARs)

\section{Background}

Gouty arthritis (GA) has recently been occurring more frequently in China and worldwide [1]. It is common in men compared to women [2]. This disorder results from high serum uric acid concentrations; monosodium urate (MSU) crystals are subsequently formed and deposited in both joints and tissues. Symptoms occur during acute episodes, and extreme pain is noted in this period [3].

\footnotetext{
* Correspondence: zhouqijoejoe@163.com

${ }^{1}$ Research Institute of Chinese Medicine, Heilongjiang University of Chinese Medicine, Harbin 150040, P.R. China

Full list of author information is available at the end of the article
}

Comorbidities are common in gout patients, such as arrhythmias, obesity, diabetes mellitus, and chronic kidney disease $[4,5]$. Current drug used to treat GA may have some side effects which could be seen obviously from our previous study, such as stomach injury by indomethacin. It is urgent to identify new drugs to treat GA since it was detrimental to the health of the patients and especially in the condition of comorbidities [6]. Mitogen-activated protein kinases (MAPKs) play an important role during the pathogenesis of GA [7]. MAPKs are classified into main three types: extracellular signal-regulated kinase-1/2 (ERK1/2), c-Jun N-terminal 
kinases (JNKs) and p38 MAPKs. MAPKs activate the transcription factors NF- $\mathrm{KB}$ and $\mathrm{AP}-1$ and regulate the expression of inflammatory factors, adhesion factors and chemokines. Peroxisome proliferator-activated receptors (PPARs) are useful in the treatment of a series of diseases such as type 2 diabetes mellitus, dyslipidaemia and cardiovascular diseases $[8,9]$. Among them, PPAR $\gamma$ is especially relevant to GA. PPAR $\gamma$ competitively inhibits NF- $\mathrm{kB}$ and AP-1 activity. A PPAR $\gamma$ agonist has been effectively used to treat GA [10]. In addition, adiponectin (ADP) is a type of protein hormone that is secreted by fat cells. Its receptors are classified to three types, including AdipoR1, AdipoR2 and T-cadherin. ADP increases the sensitivity of insulin, exhibits antiinflammatory effects, and protects the endothelium. PPAR $\gamma$ agonists promote increased expression of ADP and AdipoR2, which potentially serve as new targets to treat GA [11]. Drugs that are clinically used to treat GA exhibit various side effects. Gout drugs increase risk of the most common cancers, particularly in leukemia, non-Hodgkin's, endometrial, breast and cervical cancer [12].. Rhizoma Dioscorea Nipponicae (RDN) is the rhizome of Dioscorea nipponica Makino that belongs to the Diocoreaceae family and is mainly distributed in the Northeast, North, West, and Qinghai regions of China. As an important traditional herb, this medicine has a long history of use in China [13]. This traditional medicine is utilized by a large number of individuals $[14,15]$. Its major compound is steroidal saponin and RDN effectively reduces uric acid levels and exhibits antiinflammatory effects. RDN is being explored as a new drug alone and in combination with other Western drugs in the treatment of GA $[16,17]$.

Our previous study showed that RDN could treat GA by interfering with the NALP3 inflammasome and the TLR2/4-IL-1R signal pathway, in this way, it influenced the expression and activities of inflammatory factors [18, 19]. Given that the MAPK-PPAR $\gamma$ is another branch down- stream signal pathway of TLR2/4-IL-1R that could influence the activities of transcriptional factors and thus regulates the expression of inflammatory factors, adhesion factors and chemokines, we assumed that it may also influence this signal pathway and had approved this by an in vitro study [20]. This study aims to further investigate the effect of RDN on interfering with the MAPK-PPAR $\gamma$ signalling pathway to treat GA by using animal models. In this study, we used MSUinduced Wistar rats as the GA models which was reported in a previously published paper [21]. HE staining was used to detect the histopathologic changes of the synovial tissue of joint in order to make sure the models were built successfully. The expression of the key protein factors involved in this signalling pathway by immunohistochemistry. PPAR $\gamma$ and Adipo 2 mRNA and protein expression were also assessed by real-time PCR and WB methods. Serum chemokine (C-X-C motif) ligand protein CXCL1 and ADP levels were measured using the ELISA method. Our results showed that RDN reduces MKK4, p-MEK1/2, p-JNK, p-ERK1/2, and ICAM1 protein expression and increases PPAR $\gamma$ mRNA and protein expression. RDN also reduces serum CXCL1 levels and increases serum ADP levels. These results demonstrate that the MAPK-PPAR $\gamma$ signalling pathway is an effective target for the treatment of GA.

\section{Methods}

\section{Chemicals and reagents}

MSU was purchased from Sigma-Aldrich (St. Louis, USA), product No. was U 2875. ELISA test.

kits for CXCL1(DRE21465) and ADP (DRE 20701) were purchased from Tianjin Zombio Science and Technology Co., Ltd. (TianJin, China). Crude RDN crude drug extracts were purchased from the Harbin World Electuary Factory, product number was 090501. Colchicine (Purity rate $>99$ ) was purchased from Xishuangbanna Pharmaceutical Co., Ltd. (Xishuangbanna, China, 151,121). RNA extraction (BK5106), reverse transcription (BK1401), and SYBR Green kits (BK1504) were purchased from Dalian Bao Biotechnology (Dalian, China). PMSF mother liquor (0754) and BSA (0332) were purchased fromAmresco (Washington, America). NC membrane was purchased from Millipore (Massachusetts, America).

\section{Plant material and extraction}

RDN was purchased from the Heilongjiang Province Drug Company. The voucher specimens (hlj-201,508) of the herb were authenticated by Prof. Wang Zhen Yue, Hei long Jiang University of Chinese Medicine. One gram of crude drug was extracted with $6 \mathrm{~mL} \mathrm{50 \%} \mathrm{etha-}$ nol for $1.5 \mathrm{~h}$ for three times and purified by D-101 macroporous adsorption resin as described previously [18]. The extraction ratio was $4.97 \%(w / w)$.

\section{Determination of Rhizoma Dioscorea Nipponica content}

The calculated content of RDN in the extract was approximately $55.9 \%$ [22].

\section{UPLC/MS analysis}

UPLC/MS analysis was performed to chemically standardize the herbal extract.

Three major compounds in the RDN extract were identified: dioscin, protodioscin and pseudo protodios$\operatorname{cin}[22]$.

\section{Animals}

We purchased sixty male Wistar rats $(200 \pm 20 \mathrm{~g})$ from Shenyang Changsheng Biotechnology Co., Ltd. (China). 
They were raised in the animal house of the Institute of Chinese medicine, Heilongjiang University of Chinese Medicine and allowed 7 days to adapt to the environment before experiments.

Ten rats were housed per cage $(600 \times 450 \times 280 \mathrm{~mm})$ with the light on for $12 \mathrm{~h}$ and off for another $12 \mathrm{~h}$ each day, which was started since 07:00 a.m. They were housed at room temperature $\left(23 \pm 2{ }^{\circ} \mathrm{C}\right)$ with relative humidity $(55 \pm 5 \%)$ and given a standard chow and water ad libitum. To protect the animals, ethical approval for the experiments was obtained based on the Legislation on the Protection of Animals Used for Experiment Purposes (Directive 86/609/EEC). The Institutional Animal Care Committee approved the experiment (approval number was 20,190,921).

\section{Induction of gouty arthritis in rats using MSU}

The method to induce rats of gouty arthritis were described in a previously published paper [18].

\section{Drug administration}

Sixty male Wistar rats were divided into 6 groups randomly: The normal group, model group, groups treated with total saponin at a high dose $(160 \mathrm{mg} / \mathrm{kg})$, middle dose $(80 \mathrm{mg} / \mathrm{kg})$ and low dose $(40 \mathrm{mg} / \mathrm{kg})$ as well as colchicine group (COL) $(0.3 \mathrm{mg} / \mathrm{kg})$. The doses used was calculated according to clinical use [18]. All the drug groups were given every $24 \mathrm{~h}$ for 7 days. The normal group and the model group were administered normal saline as a control. MSU was used to induce the model of GA continuously for 5 days.

\section{Blood and synovial tissue sample collection}

Whole blood and synovial samples were collected 1 hour after the model was induced on the seventh day as previously described. All efforts were made to minimize suffering. Before tissue collection, rats were deeply anesthetized by intraperitoneal injection of $3 \%$ pentobarbital sodium $(45 \mathrm{mg} / \mathrm{kg})$. At the end of the experiments, rats were sacrificed by cervical dislocation and synovial tissues were excised and kept in $4 \%$ paraformaldehyde and liquid nitrogen before tests [23].

\section{Histopathological assessment}

Hematoxylin and eosin (HE) assessment to detect synovial tissue was described in a previously published paper [22].

Immunohistochemical analysis of P-38, p-P38, JNK, p-JNK, ERK1/2, p-ERK1/2, MEK1/2, p-MEK1/2, MKK4, p-MKK4, ICAM1 and VCAM1

Rat synovial tissue was removed and post-fixed overnight in $4 \%$ paraformaldehyde. Then, tissues were embedded in paraffin. Serial sections $(5 \mathrm{~mm})$ were collected and immunohistochemically stained for P-38, p-P38, JNK, p-JNK, ERK1/2, p-ERK1/2, MEK1/2, pMEK1/2, MKK4, p-MKK4, PPAR $\gamma$, ICAM1 and VCAM1 using the high-pressure antigen repair method according the kit's instructions. Primary antibodies included antirat P-38 antibody, anti-rat p-P38 antibody, anti-rat JNK antibody, anti-rat $\mathrm{p}-\mathrm{JNK}$ antibody, anti-rat ERK1/2 antibody, anti-rat p-ERK1/2 antibody, anti-rat MEK1/2 antibody, anti-rat p-MEK1/2 antibody, anti-rat MKK4 antibody, anti-rat p-MKK4 antibody, anti-rat PPAR $\gamma$ antibody, anti-rat ICAM1 antibody and anti-rat VCAM1 antibody (all of these antibodies were raised in rabbit, 1 : 200). Each slide was examined at 200X magnification using an Olympus BX60 Microscope (Japan). All the antibodies used are described in Table 1 (Supplementary material).

\section{Real-time PCR of PPARy and AdipoR2}

RNA was extracted using the classical TRIzol reagent method. The sequences of the gene-specific PCR primers and the lengths of the products are summarized in Table 2 (Supplementary material). Amplification was performed as previously described [18].

\section{Western blotting of PPAR $y$ and AdipoR2}

Western blotting was performed as previously described [18]. The specific antibodies used included rPPARY (1: 2000), rAdipoR2 (1:20000) and rGAPDH (1:2000) (Table 1).

Table 1 Summary of antibodies used in Immunohistochemical analysis and WB analysis

\begin{tabular}{llll}
\hline Company & Method & Description & Catalog number \\
\hline Abcam & Immunohistochemical & P-38 & ab27986 \\
& p-P38 & ab3838 \\
& JNK & ab76125 \\
& p-JNK & ab219584 \\
& ERK1/2 & ab17942 \\
& p-ERK1/2 & ab17942 \\
& MEK1/2 & ab131517 \\
& p-MEK1/2 & ab194754 \\
& MKK-4 & ab131494 \\
& p-MKK-4 & ab131494 \\
& PPARY & ab209350 \\
& ICAM1 & ab2213 \\
Bioss & VCAM1 & ab134047 \\
& PPARY & BS-0530R \\
Immunoway & AdipoR2 & BS-0611R \\
& GAPDH & YM3029 \\
\hline
\end{tabular}


Table 2 Summary of Gene-Specific Real-Time PCR Primer Sequences

\begin{tabular}{llll}
\hline Description & Gene bank & Sense primer (5'-3') & Anti-sense primer $\left(3^{\prime}\right.$-5') \\
\hline PPARY & NM_013124 & TAGGTGTGATCTTAACTGTCG & GCATGGTGTAGATGATCTCA \\
AdipoR2 & NM_001037979 & ATGTTTGCCACCCCTCAGTA & CAGATGTCACATTTGCCAGG \\
GAPDH & NM_008084 & TGCACCACCAACTGCTTAC & GATGCAGGGATGATGTTC \\
\hline
\end{tabular}

\section{ELISA tests for CXCL1 and ADP}

CXCL1 and ADP blood levels were assessed using kits.

\section{Statistical analysis}

All data are expressed as the mean \pm standard error of the mean (S.E.M.), and statistical analysis was performed using a one-way analysis of variance (ANOVA) followed by Dunnett's t-test to determine the level of significance. A $P$-value $<0.05$ was considered statistically significant.

\section{Results}

Histopathological analysis of rat synovial tissue

As shown in Fig. 1, synovial tissue was obtained from the knee joint 7 days after MSU injection. In the control group, synoviocytes formed a monolayer. No abnormal inflammatory cells were observed in synovial tissue. Light microscopic evaluation demonstrated a serious inflammatory reaction in GA rats. Specifically, synovial hyperplasia was obvious, and cells were irregularly arranged. In addition, inflammatory cell infiltration and pannus formation were observed. In contrast, synovial tissue damage in the $\mathrm{RDN}$ and COL groups was less severe. In addition, synovial hyperplasia was inhibited, and the inflammatory response was reversed.

P38, p-P38, JNK, p-JNK, ERK1/2, p-ERK1/2, MEK1/2, pMEK1/2, MKK4, p-MKK4, VCAM1 and ICAM1 protein expression as determined by Immunohistochemical analysis

As shown in Figs. 2 and 3a, b and c, P38, p-P38 and JNK levels in the control group were $0.122 \pm 0.017,0.146 \pm$ 0.021 and $0.153 \pm 0.031$, respectively. The respective levels in the model group were $0.134 \pm 0.008,0.141 \pm$ 0.017 and $0.187 \pm 0.017$. No statistical differences were noted compared with control group. Compared with the model group, P38, p-P38 and JNK levels were $0.151 \pm$ $0.004,0.158 \pm 0.013$ and $0.185 \pm 0.032$ in the high-dose group; $0.123 \pm 0.015,0.170 \pm 0.017$, and $0.178 \pm 0.007 \mathrm{in}$ the middle-dose group; $0.124 \pm 0.006,0.151 \pm 0.012$ and $0.188 \pm 0.016$ in the low-dose group; and $0.148 \pm 0.017$, $0.146 \pm 0.029$ and $0.193 \pm 0.040$ in the COL group. No statistically significant differences were noted compared with the model group.
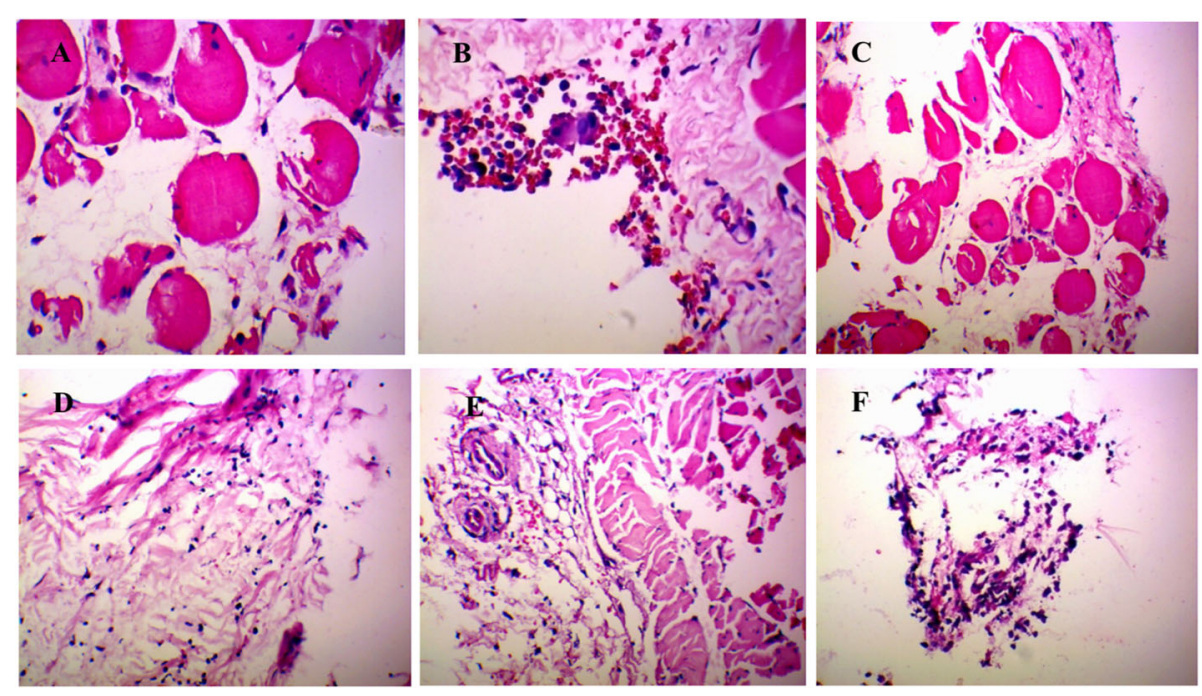

Fig. 1 Histopathological analysis of rat synovium tissue. a Control group and $\mathbf{b}$ model group were treated with saline for 5 days. c High dose group $\mathbf{d}$ middle dose group and e low dose group were treated with extract of RDN for 5 days, dose amounted to $40 \mathrm{mg} / \mathrm{kg}, 80 \mathrm{mg} / \mathrm{kg}$ and 160 $\mathrm{mg} / \mathrm{kg}$ daily, respectively. $\mathbf{f} \mathrm{COL}$ group was treated with COL for 5 days. Each slide was examined at a magnification of 200 times. Histopathological changes of H\&E-stained sections were used to assess the severity of GA, characterized by infiltration of inflammatory cells and synovium swelling and distention as well as vasocongestion and tissue necrosis. In contrast, healthy synovium showed almost no inflammatory cells 


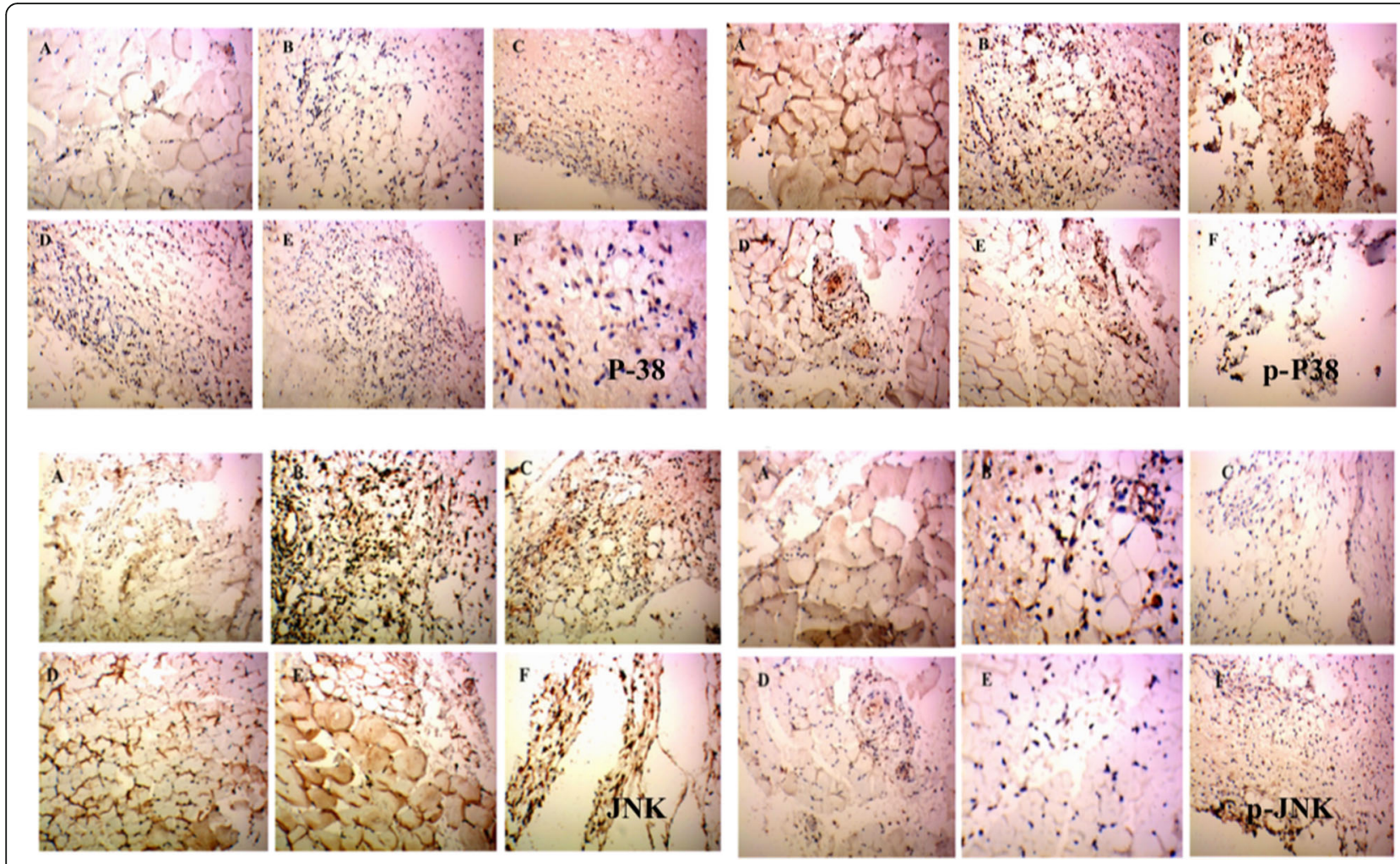

Fig. 2 Immunohistochemical staining of P38, p-P38, JNK and p-JNK in rat synovium tissue. (A) Control group and (B) model group were treated with saline for 5 days. (C) High dose group (D) middle dose group and (E) Low group were treated with extract of RDN for 5 days, and dose amounted to $40 \mathrm{mg} / \mathrm{kg}, 80 \mathrm{mg} / \mathrm{kg}$ and $160 \mathrm{mg} / \mathrm{kg}$ daily, respectively. Each slide was examined at a magnification of 200 times and purple P38, pP38, JNK and p-JNK positive cells can be found within rat synovium tissue. Images shown are a representative from three independent trials. $\# \#<0.001$ vs control group (independent samples $t$-test), ${ }^{* * *} p<0.001$ vs model group (one-way ANOVA followed by Dunnett's $t$-test)

As shown in Figs. 2 and 3d, p-JNK levels were $0.154 \pm$ $0.015,0.413 \pm 0.064,0.145 \pm 0.019,0.145 \pm 0.019,0.115$ \pm 0.010 , and $0.143 \pm 0.007$ in the control, model, RDN high, RDN middle, RDN low and COL groups. Compared with control group, p-JNK protein levels were increased by $168.2 \%(P<0.001)$ in the model group. Compared with model group, p-JNK protein levels were decreased by $64.9 \%(P<0.001), 64.9 \%(P<0.001), 72.2 \%$ $(P<0.001)$ and $65.4 \%(P<0.001)$ in the RDN high, RDN middle, RDN low and COL groups.

As shown in Figs. 3e and 4, ERK1/2 levels were 0.150 $\pm 0.22,0.158 \pm 0.012,0.148 \pm 0.017,0.173 \pm 0.028,0.153$ \pm 0.011 , and $0.189 \pm 0.012$ in the control group, model group, RDN high group, RDN middle group, RDN low group and COL group, respectively. No significant difference was noted among these groups.

As shown in Figs. 3f and 4, p-ERK1/2 levels were $0.141 \pm 0.020,0.207 \pm 0.018,0.143 \pm 0.017,0.164 \pm$ $0.008,0.131 \pm 0.007$, and $0.184 \pm 0.012$ in the control, model, RDN high, RDN middle, RDN low and COL groups, respectively. Compared with the control group, p-ERK $1 / 2$ protein levels were increased by $46.8 \%$ in the model group $(P<0.01)$. Compared with the model group, p-ERK1/2 protein levels were decreased by $30.9 \%$ $(P<0.05), 20.8,36.7 \%(P<0.01)$ and $11.1 \%$ in the RDN high, middle, low and COL groups.

As shown in Figs. 4 and 5a, MEK1/2 levels were 0.149 $\pm 0.29,0.201 \pm 0.025,0.174 \pm 0.020,0.154 \pm 0.021,0.159$ \pm 0.007 , and $0.204 \pm 0.014$ in the control, model, RDN high, RDN middle, RDN low and COL groups, respectively. No significant differences were noted among all these groups.

As shown in Figs. 4 and 5b, p-MEK $1 / 2$ levels were $0.170 \pm 0.033,0.309 \pm 0.046,0.206 \pm 0.019,0.169 \pm$ $0.022,0.177 \pm 0.016$, and $0.198 \pm 0.010$ in the control, model, RDN high, RDN middle, RDN low and COL group. Compared with control group, $\mathrm{p}-\mathrm{MEK} / 2$ protein levels were increased by $81.7 \%$ in the model group $(P<$ $0.01)$. Compared with the model group, p-MEK $1 / 2$ protein levels were decreased by $33.3 \%(P<0.05), 45.3 \%$ $(P<0.01), 42.7 \%(P<0.01)$ and $35.9 \%(P<0.05)$ in the RDN high, RDN middle, RDN low and COL groups.

As shown in Figs. 5c and 6, MKK4 levels were $0.165 \pm$ $0.013,0.229 \pm 0.023,0.236 \pm 0.024,0.157 \pm 0.010,0.179$ 

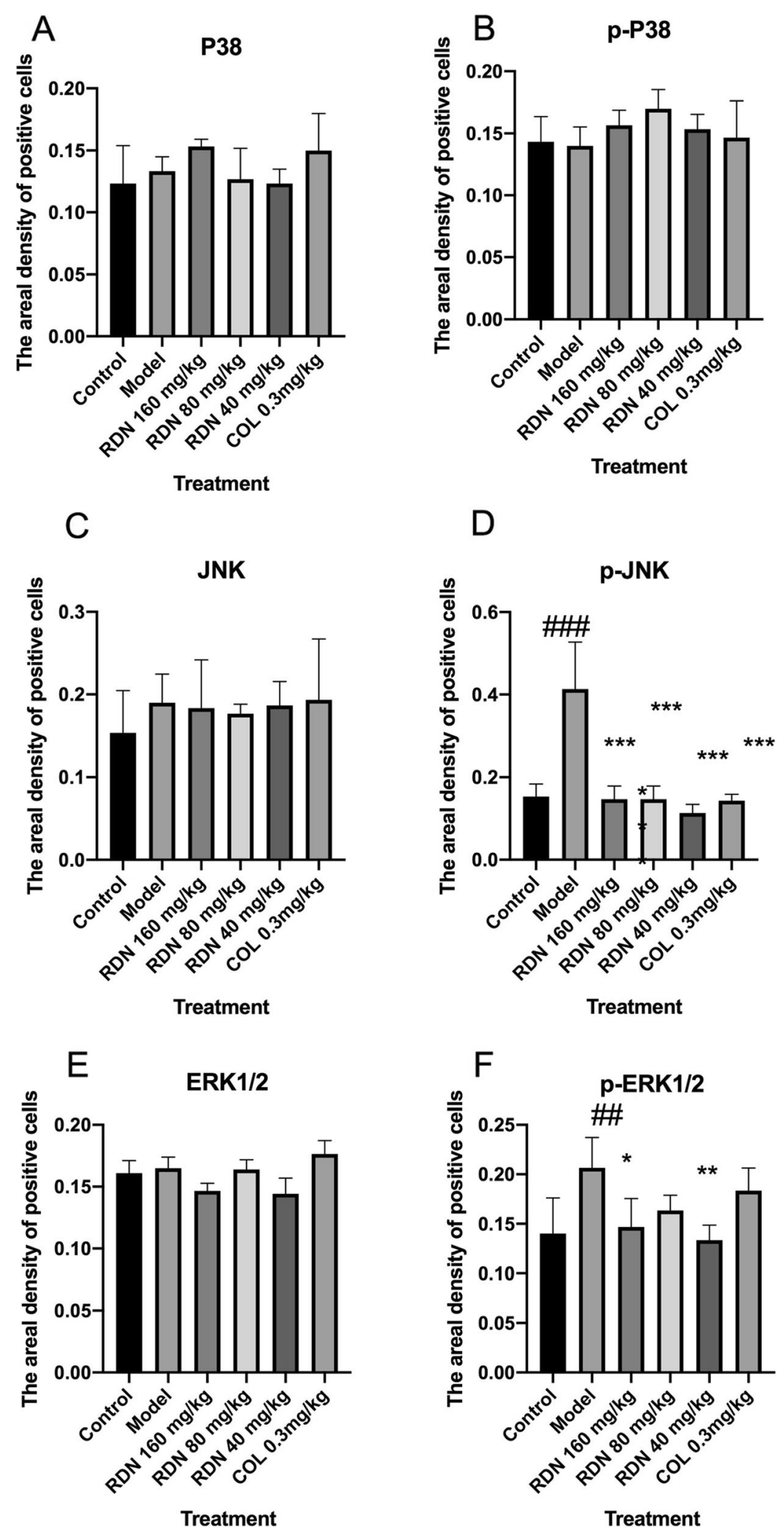

Fig. 3 (See legend on next page.) 
(See figure on previous page.)

Fig. 3 The levels of P38, p-P38, JNK, p-JNK, ERK1/2 and p-ERK1/2 in the rat synovium tissue. The areal densities of P38, p-P38, JNK, p-JNK, ERK1/2 and p-ERK1/2-positive cells in full image were calculated by Motic Med 6.0 Digital Medical Image Analysis System. Data represent the mean SE of each group $(n=10) .{ }^{\# \#} p<0.01$ vs control group; ${ }^{\# \# \#} p<0.001$ (independent samples $t$-test), $p<0.05$ vs model group; $p<0.01$ vs model group; ${ }^{* * *} p<0.001$ (one-way ANOVA followed by Dunnett's $t$-test)

\pm 0.015 , and $0.183 \pm 0.009$ in the control, model, RDN high, RDN middle, RDN low and COL groups. Compared with the control group, MKK4 protein levels were increased by $38.8 \%$ in the model group $(P<0.05)$. Compared with the model group, MKK4 protein levels were increased by $3.05 \%$ in the RDN high group. Compared with the model group, MKK4 protein levels were decreased by $31.4 \%(P<0.05), 21.8 \%(P<0.05)$ and $20.1 \%$ in the RDN middle, RDN low and COL groups.

As shown in Figs. 5d and 6, p-MKK4 levels were $0.185 \pm 0.39,0.201 \pm 0.027,0.207 \pm 0.037,0.175 \pm$ $0.016,0.153 \pm 0.004$, and $0.204 \pm 0.014$ in the control, model, RDN high, RDN middle, RDN low and COL groups. No significant differences were noted among all these groups.
As shown in Figs. 5e and 6, ICAM1 levels were 0.104 $\pm 0.014,0.166 \pm 0.006,0.161 \pm 0.038,0.139 \pm 0.013$, $0.108 \pm 0.006$, and $0.128 \pm 0.003$ in the control, model, RDN high, RDN middle, RDN low and COL groups. Compared with the control group, ICAM1 protein levels wereincreased by $59.6 \%$ in the model group $(P<0.05)$. Compared with the model group, ICAM1 protein levels were decreased by $3.01,16.3,34.9 \%(P<0.05), 35.9$ and 20.1\%in the RDN middle, RDN low and COL groups.

As shown in Figs. 5f and 6, VCAM1 levels were $0.102 \pm 0.018,0.149 \pm 0.011,0.159 \pm 0.038,0.144 \pm$ $0.021,0.143 \pm 0.010$, and $0.139 \pm 0.006$ in the control, model, RDN high, RDN middle, RND low and COL groups. No significance differences were noted among all these groups.

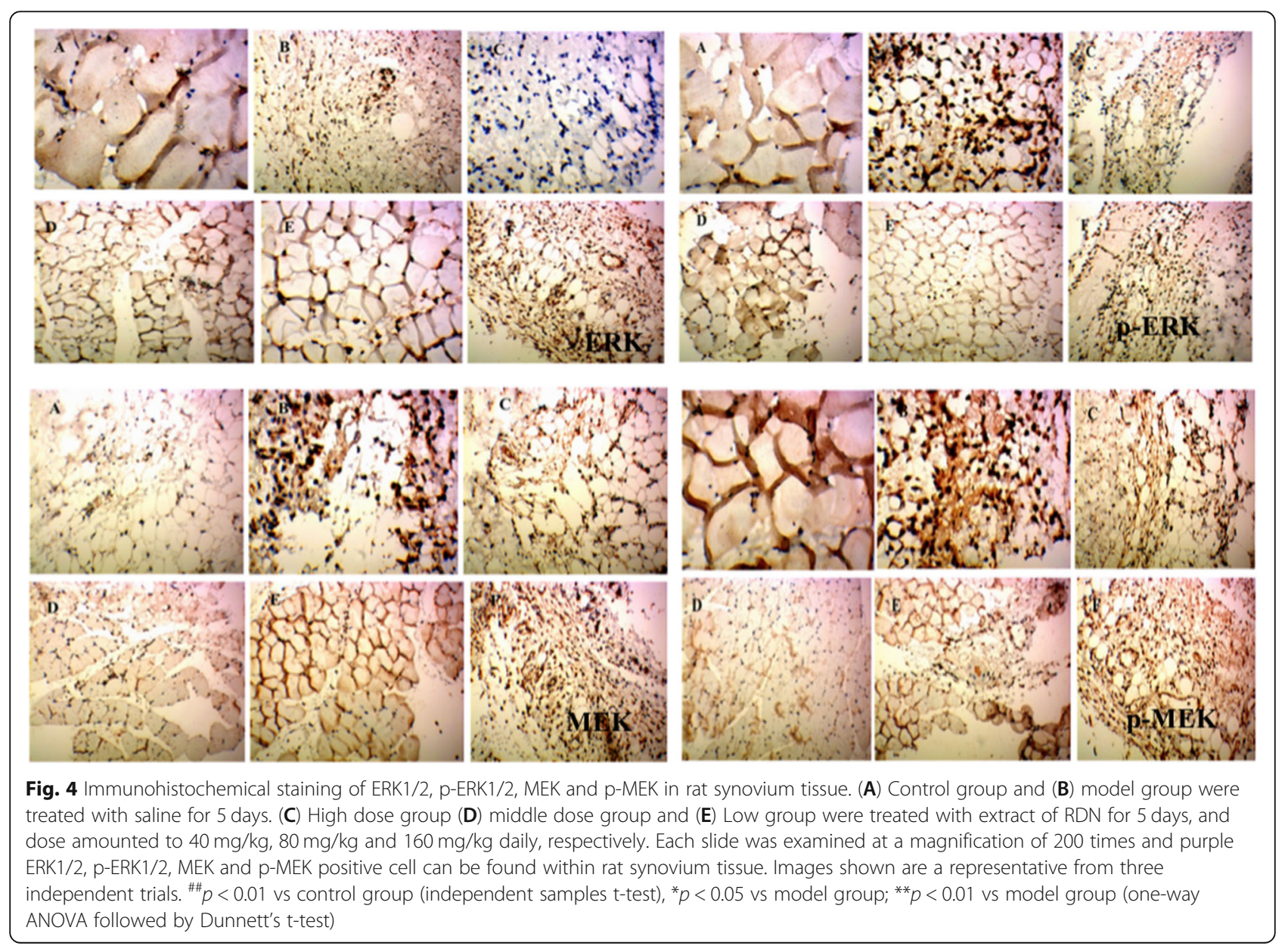



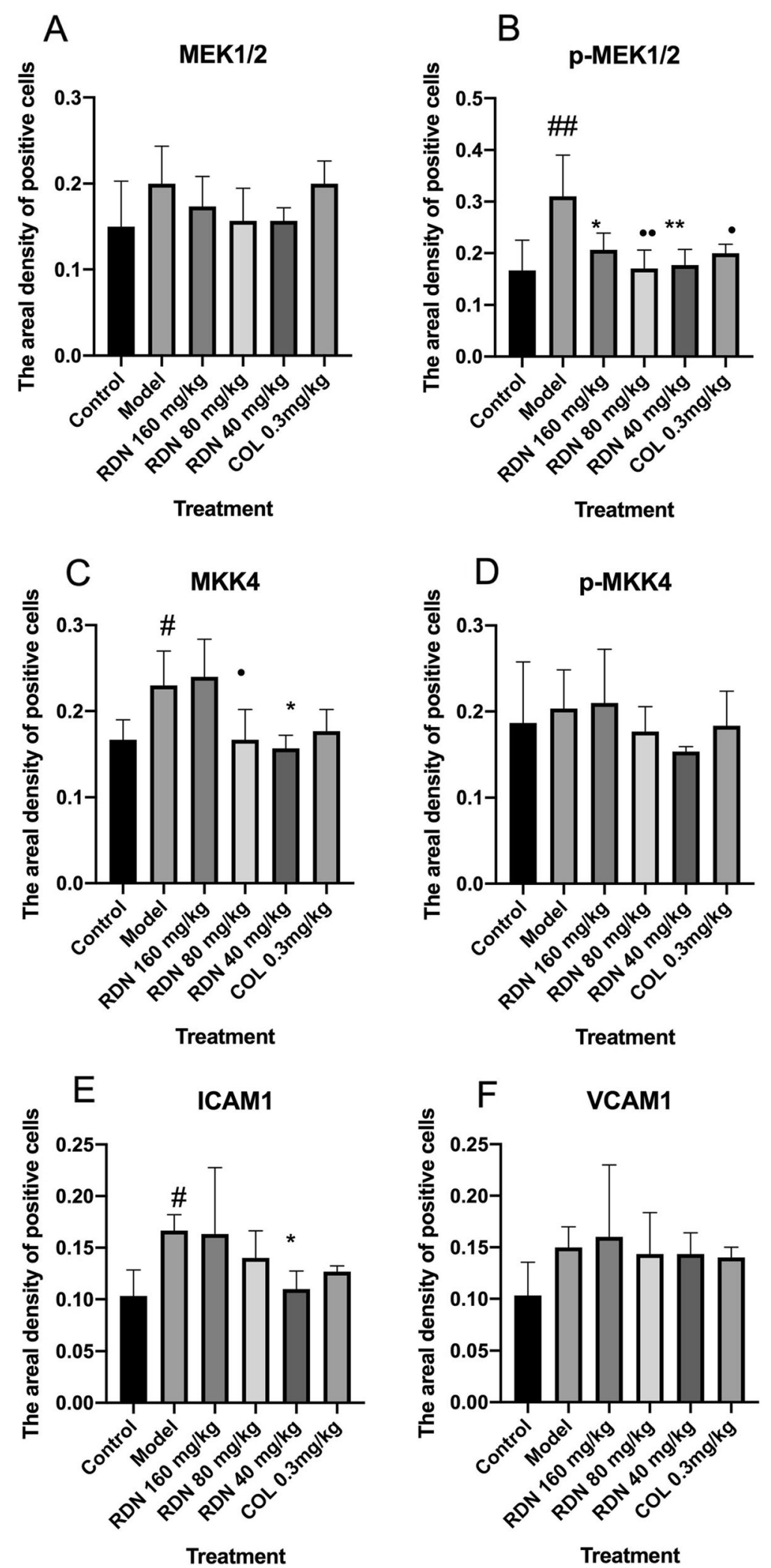

Fig. 5 (See legend on next page.) 
(See figure on previous page.)

Fig. 5 The levels of MEK1/2, p-MEK1/2, MKK4, p-MKK4, ICAM1 and VCAM1 in the rat synovium tissue. The areal densities of MEK1/2, p-MEK1/2, MKK4, p-MKK4, ICAM1 and VCAM1-positive cells in full image were calculated by Motic Med 6.0 Digital Medical Image Analysis System. Data represent the mean SE of each group $(n=10)$. ${ }^{\#} p<0.05$ vs control group, ${ }^{\# \#} p<0.01$ (independent samples $t$-test), ${ }^{*} p<0.05$ vs model group; ${ }^{* *} p<$ 0.01 vs model group (one-way ANOVA followed by Dunnett's $t$-test)

\section{Effects of RDN on PPAR $y$ and AdipoR2 mRNA and protein expression}

As shown in Table 3, Fig.7a, b, c, and d, and Fig. 8, Compared with the normal group, PPAR $\gamma$ mRNA expression was increased $(p<0.001)$, they were all decreased in the RDN high, middle, low dose groups and COL group $(p<0.001, p<0.01, p<0.05$ and $p<$ $0.05)$. Compared with the normal group, the protein expression in the model group was decreased significantly $(p<0.05)$. Compared with the model group, the expressions of PPAR $\gamma$ were decreased significantly in the high, middle and COL groups $(p<0.01, p<0.01$, and $p<0.01)$.

Compared with the normal group, the mRNA expression of AdipoR2 in the model group was increased significantly $(p<0.01)$. Compared with the model group, the mRNA expression of AdipoR2 in the RDN high, low group and COL group were decreased significantly $(p<$ $0.05, p<0.05$, and $p<0.05)$. However, there was no difference of AdipoR2 protein expression among these groups.

\section{Effects of RDN on CXCL1 and ADP levels in MSU crystal- induced rats}

As shown in Fig. 9a and b, compared with the normal group, CXCL1 levels were increased significantly $(p<$ $0.001)$ in the model group. The high $(160 \mathrm{mg} / \mathrm{kg})$ dose, middle $(80 \mathrm{mg} / \mathrm{kg})$, and low $(40 \mathrm{mg} / \mathrm{kg}) \mathrm{RDN}$ doses and COL dose significantly reduce CXCL1 levels $(p<0.001$, $p<0.001, p<0.001$, and $p<0.001$, respectively) compared with the model group. Compared with the normal

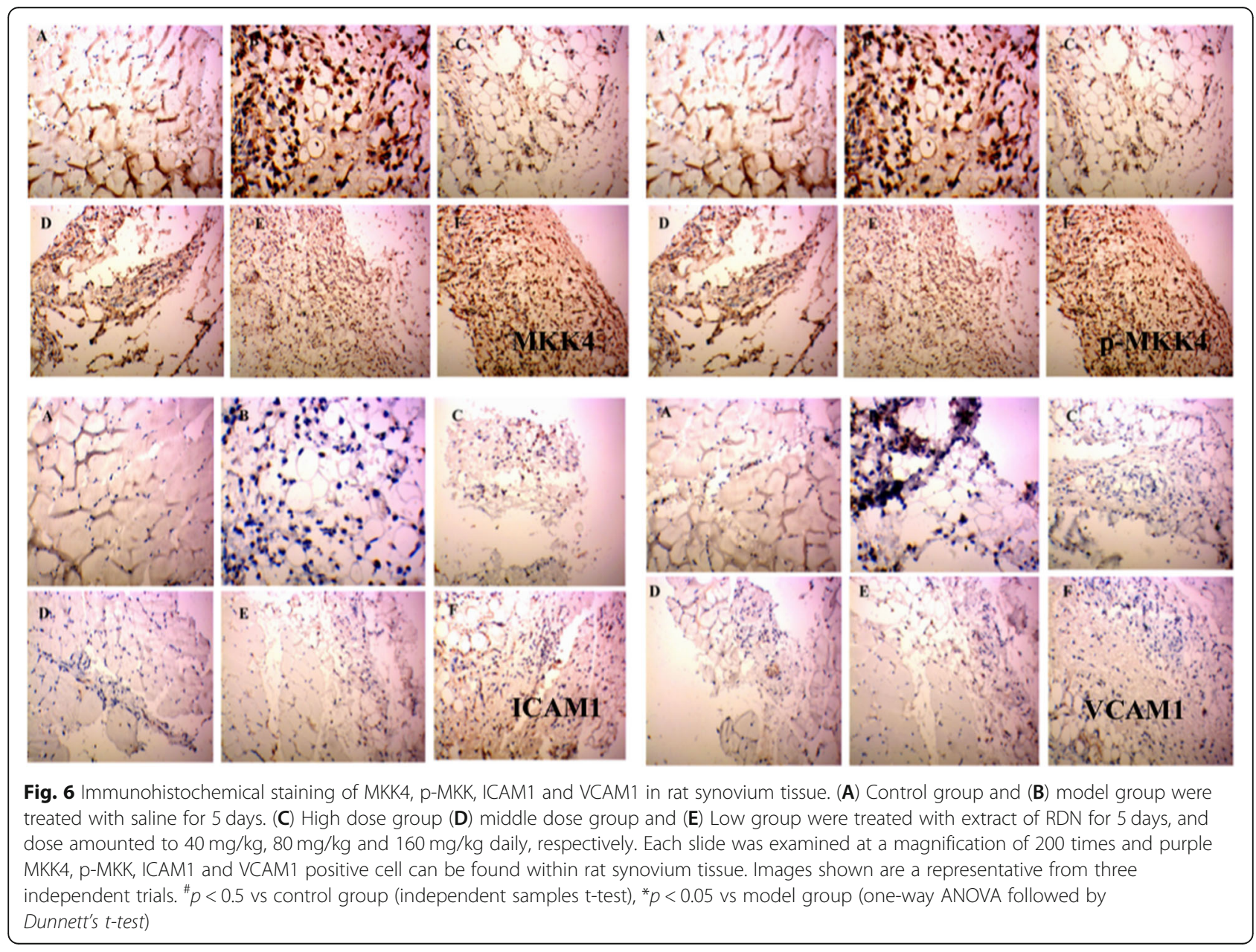


Table 3 Protein expression change of PPARy and AdipoR2

\begin{tabular}{llll}
\hline Group & Dose $(\mathrm{mg} / \mathrm{kg})$ & PPAR & AdipoR2 \\
\hline Normal & - & $0.343 \pm 0.020$ & $0.528 \pm 0.022$ \\
Model & - & $0.560 \pm 0.096^{\# \#}$ & $0.553 \pm 0.027$ \\
RDN & 160 & $0.287 \pm 0.008^{* *}$ & $0.467 \pm 0.011$ \\
RDN & 80 & $0.213 \pm 0.006^{* * *}$ & $0.469 \pm 0.022$ \\
RDN & 40 & $0.440 \pm 0.020$ & $0.520 \pm 0.040$ \\
COL & 0.3 & $0.206 \pm 0.008^{* * *}$ & $0.465 \pm 0.012$ \\
\hline
\end{tabular}

Data represent mean \pm SEM for10 rats, ${ }^{\# \#} p<0.01$ compared with normal group (independent samples t-test); ${ }^{* *} p<0.01,{ }^{* * *} p<0.001$ compared with model group (one-way ANOVA followed by Dunnett's $t$-test) group, ADP levels were decreased significantly $(p<0.01)$ in the model group. High $(160 \mathrm{mg} / \mathrm{kg})$, middle $(80 \mathrm{mg}$ / $\mathrm{kg}$ ), and low $(40 \mathrm{mg} / \mathrm{kg}) \mathrm{RDN}$ doses and the COL dose significantly increase ADP levels $(p<0.001, p<0.001$, $p<0.01$, and $p<0.01$, respectively) compared with the model group.

\section{Discussion}

GA is a disease that is associated with a difficult recovery, since its symptoms only manifest during the attack period and the chronic period when high concentrations of uric acid are present in the blood [5]. Dioscorea

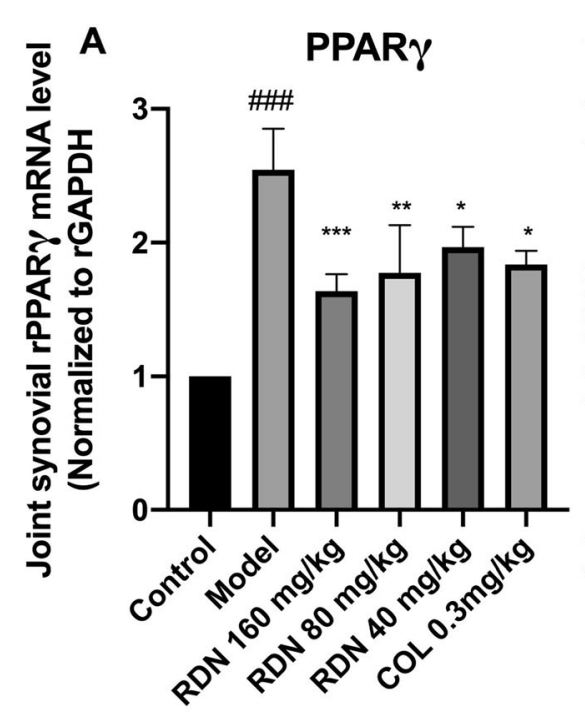

Treatment

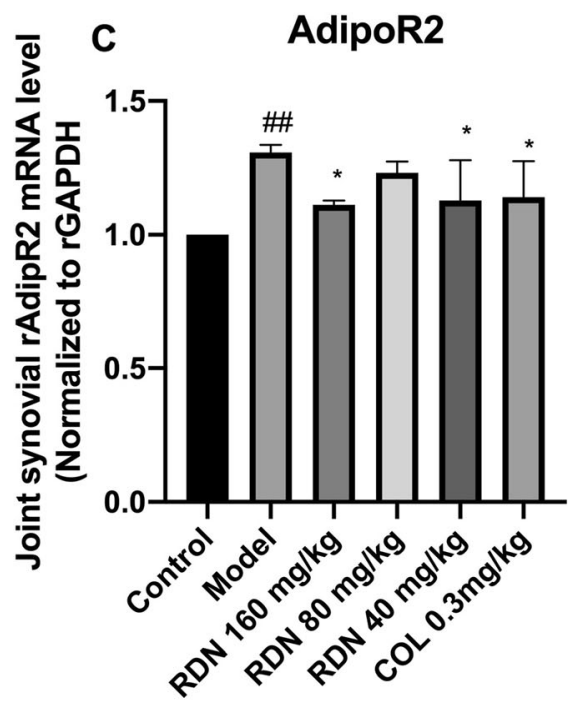

Treatment
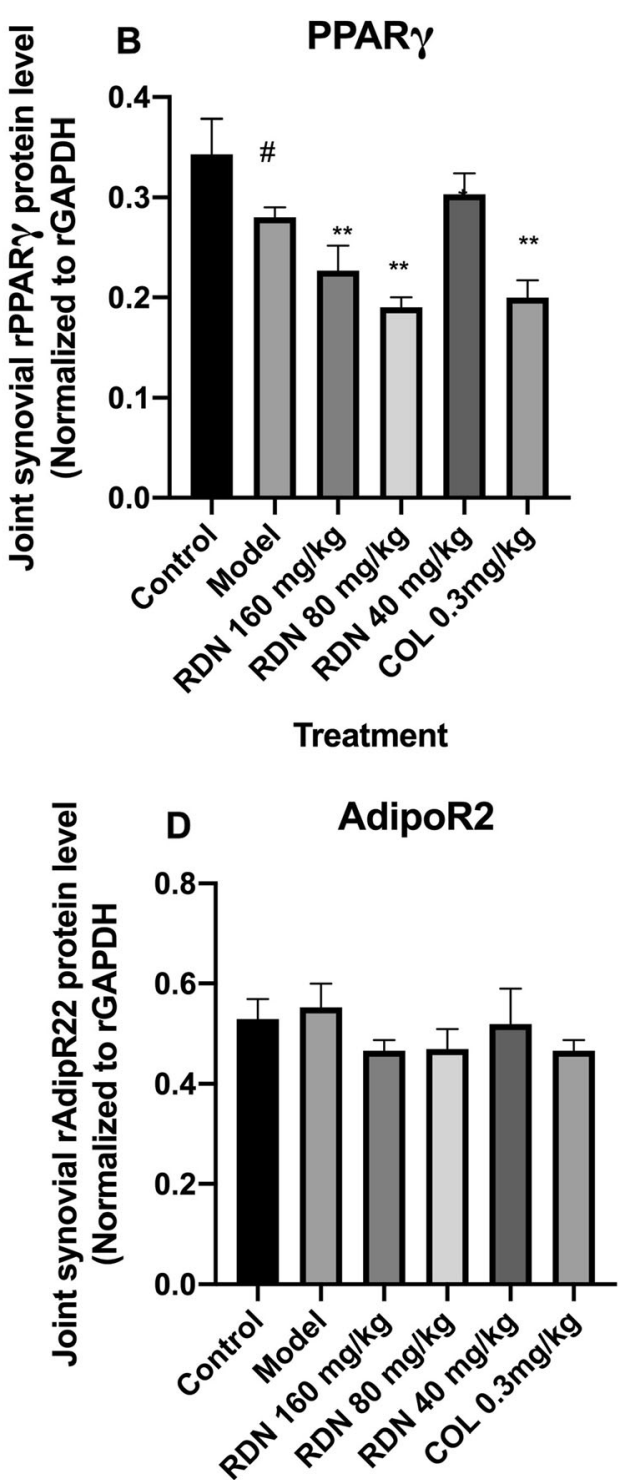

Treatment

Fig. 7 Effects of RDN and COL on mRNA and protein levels of PPARY (a and $\mathbf{b}$ ) and AdipoR2 (c and $\mathbf{d}$ ) in the joint-synovial tissue in GA rats. The data represent the values of the mean \pm S.E.M. for six mice. ${ }^{\# \#} P<0.01$, ${ }^{\# \#} P<0.001$ versus normal group (independent samples t-test). ${ }^{*} P<0.05$, ${ }^{* *} P<0.01,{ }^{* * *} P<0.001$ versus model group (one-way ANOVA followed by Dunnett's $t$-test) 


\section{$\begin{array}{llllll}1 & 2 & 3 & 4 & 5 & 6\end{array}$

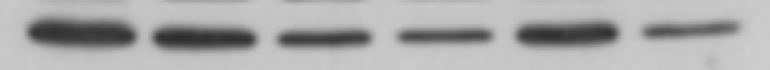 \\ rPPAR $\gamma$

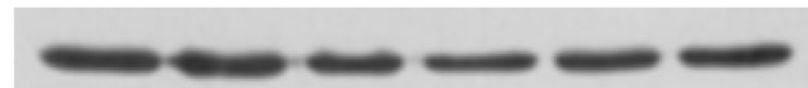 \\ rAdiprR2 \\ $57 \mathrm{kDa}$ \\ $44 \mathrm{kDa}$ \\ $37 \mathrm{kDa}$}

Fig. 8 Effects of RDN and COL on the protein levels of rPPARY and rAdipoR2 in the joint-synovial tissue of GA rats. RDN and COL were given 3 days before the models were induced every $24 \mathrm{~h}$. The normal group and the model group were given normal saline at the same time. MSU solution was injected into both knees after intraperitoneal injection of $10 \%$ chloral hydrate to anaesthetize the rats an hour after the drugs were given since the third day. The drugs were given for 7 days continuously while the models were induced for 5 days altogether. 1.Normal group, 2 . Model group,3. RDN high group, 4. RDN middle group, 5. RDN low group, 6. COL group

nipponica Makino is a traditional Chinese medicine that is clinically used to treat GA. However, the mechanism by which this drug acts against this disease is not completely understood. We have performed considerable research to reveal this mystery. Our previous study confirmed that the total saponins content of RDN was approximately $55 \%$. Its main components, including dioscin, protodioscin, and pseudo protodioscin were identified by UPLC-MS [22]. In addition to its ability to reduce urate acid levels, it also has anti-inflammatory effects $[18,19,22]$ Our newly published paper showed that RDN was effective in the treatment of GA by modulating the MAPK-PPAR $\gamma$ signaling pathway [24] However, it was an in vitro study that used fibroblastlike synoviocytes induced with IL-1 $\beta$. The purpose of this study was to assess this mechanism using a GA rat model.

RDN regulates the abnormal expression of MKK4, pMEK1/2, p-JNK, p-ERK1/2 and ICAM1 protein and PPAR $\gamma$ mRNA and protein in the synovial joint tissue. RDN reduced MKK4, p-MEK1/2, p-JNK, p-ERK1/2, and ICAM1 protein expression and decreased PPAR $\gamma$ mRNA and protein expression. RDN reduced serum CXCL1 levels and increases serum ADP levels showing that the MAPK signalling pathway is an effective target in GA. However, P38, p-P38, ERK1/2, JNK, p-MKK4 MEK1/2, VCAM1 and AdipoR2 protein expression levels did not obviously differ compared with the normal group.

MAPKs, including JNK, P38 and ERK1/2, are leading factors in GA attacks. Mitogen-activated protein kinase kinase4/7 (MKK4/7), mitogen-activated extracellular signal-regulated kinase1/2 (MEK1/2) and MKK3/6 are upstream kinases of JNK, ERK1/2 and P38, respectively [25-27]. In this study, RDN reduced the abnormal expression of MKK4, pMEK1/2, p-JNK and p-ERK1/2.
This result is consistent with another study demonstrating that kinsenoside attenuates osteoarthritis by inhibiting the MAPK signalling molecules p-JNK, p-ERK and p-P38 [19].

A PPAR $\gamma$ agonist effectively inhibits the production of inflammatory factors in the treatment of GA rats [28]. A PPAR $\gamma$ agonist also promoted increased expressions of ADP and AdipoR2 [11]. In this study, PPAR $\gamma$ mRNA and protein expression is increased in the model group compared with the normal group. RDN decreased PPAR $\gamma$ mRNA and protein expression and increased serum ADP levels. RDN also decreas.ed. AdipoR2 mRNA levels; however, AdipoR2 protein levels did not differ compared with the control group. The result is quite consistent with our in vitro study [20]. This finding may be explained by the self-recovery features of GA. It showed that RRAR $\gamma$ is an RDN target that may have other mechanism that needs further investigation.

In GA attacks, various cell surface molecules are expressed. Among them, vascular cell adhesion molecule (VCAM)-1 and intercellular adhesion molecule (ICAM)-1 are critical [29]. When monosodium urate (MSU) induces monocyte and neutrophil granulocytes, VCAM1 activates these cells, and these activated cells enter tissues around the vasculature after adhering to vascular endothelial cells under the action of chemokines, such as $\mathrm{C}-\mathrm{X}-\mathrm{C}$ motif chemokine ligand $(\mathrm{CXCL}) 1$. In this study, RDN reduced expression of both ICAM1 and CXCL1; however, similar VCAM1 protein levels were noted in the model and control groups. It was also very interesting that the low dose of RDN showed better effect compared with the high dose group. As there were multicomponents and multi-targets of RDN to act in this 


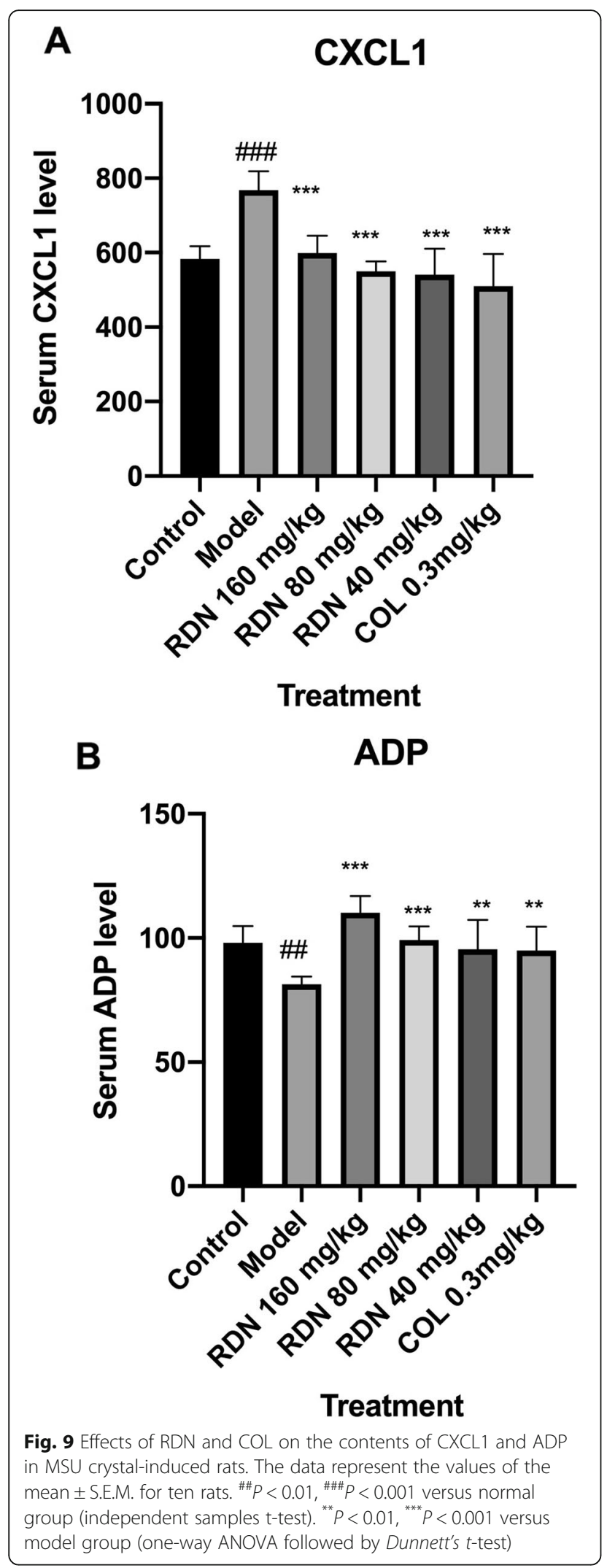

process, when one target was achieved by supersaturation, the mechanism may be changed in some aspect.

Our results showed that RDN exhibits antiinflammatory effects by modulating the MAPK/PPAR $\gamma$ signalling pathway further confirming our previous study. Since GA is a disease caused by high serum uric acid concentrations, hypertension, hyperlipidaemia and other diseases that are caused by the same mechanisms often occur as complications [30]. Chinese medicine often targets multiple targets since it has multiple components. This study showed that the MAPK/PPARy signalling pathway is a useful target in the treatment of GA. Thus, RDN should be explored as a new drug to treat GA and, especially, its complications. However, further studies to knock down or over express the key genes of this signalling pathway should be done to verify the above results.

\section{Conclusion}

RDN is potentially useful in the treatment of GA by modulating the MAPK/PPAR $\gamma$ signalling pathway. This study provides a pharmacological foundation to explore RDN as a new medicine to treat GA.

\section{Supplementary information}

Supplementary information accompanies this paper at https://doi.org/10. 1186/s12906-020-03055-7.

Additional file 1

\section{Abbreviations}

GA: Gouty arthritis; MAPK: Mitogen-activated protein kinase:

PPARs: Peroxisome proliferators-activated receptors; RK1/2: Extracellular signal regulated kinase 1/2; JNK: c-Jun N-terminal kinase; NF-kB: Nuclear factor kappa B; MKP: MAPK phosphatase; CXCL1: C-X-C motif chemokine1; VCAM-

1: Vascular cell adhesion molecule1; ICAM1: Intercellular adhesion molecular1

\section{Acknowledgements}

We thank the Chang sha Nan ke Biotechnology Co., Ltd. for technical support.

\section{Declaration}

The experimental work described in this article was conducted in the Institute of Chinese Medicine, Heilongjiang University of Chinese Medicine, Harbin, Heilongjiang, China, from October 2019 to January 2020. These studies are the result of our own investigations and have not been submitted in any other form to another journal.

\section{Authors' contributions}

QZ designed the work, HS performed the research, SL analyzed the data, XJ, QW, SZ and DY helped to interpreted the data. All authors have read and approved the manuscript.

\section{Funding}

The work was supported by Start Fund of Postdoctoral Research in Heilongjiang Province (LRB146914); Outstanding Youth Development Fund of Heilongjiang University of Chinese Medicine (2019JC06); Innovative Talent Support Programs of Colleges and Universities at the Heilongjiang Education Department (051599); Harbin Applied Technology Research and Development Project (Young Reserve Talent B, 2017RAQXJ215), National Natural Science Fund projects (81803871). The founding sponsor had no role 
in the design of the study; in the collection, analyses, or interpretation of data; in the writing of the paper; and in the decision to publish the results.

\section{Availability of data and materials}

The datasets used and analysed during the current study are available from the corresponding author upon reasonable request.

\section{Ethics approval and consent to participate}

The procedures related to the animal activities have been approved by the Departmental Animal Ethical Committee (approval number was 20190921) of Heilongjiang University of Chinese Medicine. These guidelines were in accordance of the internationally documented principles for laboratory used and care.

\section{Consent for publication}

Not applicable.

\section{Competing interests}

The authors declare that they have no competing interests.

\section{Author details}

${ }^{1}$ Research Institute of Chinese Medicine, Heilongjiang University of Chinese Medicine, Harbin 150040, P.R. China. ${ }^{2}$ Graduate School, Heilongjiang University of Chinese Medicine, Harbin 150040, P.R. China. ${ }^{3}$ Technological Innovation Team of Basic Theory Study Research of Institution of Higher Education in Heilongjiang Province, Heilongjiang University of Chinese Medicine, Harbin 150040, P.R. China.

Received: 23 May 2020 Accepted: 13 August 2020

Published online: 25 August 2020

\section{References}

1. Gupta MK, Singh JA. Cardiovascular disease in gout and the protective effect of treatments including urate-lowering therapy. Drugs. 2019;79(5): 531-41.

2. Kadiyoran C, Zengin O, Cizmecioglu HA, Tufan A, Kucuksahin O, Cure MC, et al. Monocyte to lymphocyte ratio, neutrophil to lymphocyte ratio, and red cell distribution width are the associates with gouty arthritis. Acta Med Austriaca. 2019;62(3):99-104.

3. Yang QB, He YL, Zhong XW, Xie WG, Zhou JG. Resveratrol ameliorates gouty inflammation via upregulation of sirtuin 1 to promote autophagy in gout patients. Inflammopharmacology. 2019;27(1):47-56.

4. Giannopoulos G, Angelidis C, Deftereos S. Gout and arrhythmias: in search for causation beyond association. Trends Cardiovas Med. 2019:29:41-7.

5. Robinson PC. Gout-an update of aetiology, genetics, co-morbidities and management. Maturitas. 2018;118:67-73.

6. Vazirpanah N, Ottria A, van der Linden M, Wichers CGK, Schuiveling M, van Lochem $\mathrm{E}$, et al. mTOR inhibition by metformin impacts monosodium urate crystal-induced inflammation and cell death in gout: a prelude to a new add-on therapy. Ann Rheum Dis. 2019;78(5):663-71.

7. Dalbeth $\mathrm{N}$, Haskard DO. Mechanisms of inflammation in gout. Rheumatology. 2005:44:1090-6.

8. Khan SI, Malhotra RK, Rani N, Sahu AK, Tomar A, Garg S, et al. Febuxostat modulates MAPK/NF-kBp65/TNF-a signaling in cardiac ischemia-reperfusion injury. Randomized Control Trial. 2017;2017:ID8095825.

9. Hong F, Xu P, Zhai Y. The opportunities and challenges of peroxisome proliferator-activated receptors ligands in clinical drug discovery and development. Int J Mol Sci. 2018;19(8):2189.

10. Heitel P, Gellrich L, Heering J, Goebel T, Kahnt A, Proschak E, et al. Urate transporter inhibitor lesinurad is a selective peroxisome proliferatoractivated receptor gamma modulator (sPPARyM) in vitro. Sci Rep. 2018:8(1): 13554.

11. Su J, Chai KX, Li ZQ, Cui S, Ji LH, Chen YQ, et al. Expression of adiponectin and peroxisome proliferator activated receptor $\gamma$ in patients with primary gouty arthritis and asymptomatic hyperuricemia. Qinghai Med J. 2015:45:6-10.

12. Yang HC, Nguyen PAA, Islam M, Huang CW, Poly TN, labal U, et al. Gout drugs use and risk of cancer: a case-control study. Multicenter Study. 2018; 85(6):747-53.

13. Yu H, Du JL. Pharmacological actions and mechanism of saponins from Dioscorea Nipponica. Zhongguo Zhong Yao Za Zhi. 2017;42(24):4694-9.
14. Li XZ, Zhang SN, Yang XY. Combination of cheminformatics and bioinformatics to explore the chemical basis of the rhizomes and aerial parts of Dioscorea nipponica Makino. J Pharm Pharmacol. 2017;69(12):1846-57.

15. Wu XM, Yang YY, Zhao LH. Exploration about the clinical application and dosage of Rhizoma Dioscorea Nipponicae. Jilin J Chin Med. 2019;39(2):165-8.

16. Wang $X$, Wang $Y G$. Progress in treatment of gout using Chinese and western medicine. Chin J Integr Med. 2020;26(1):8-13.

17. Qu-Yang SH, Jiang T, Zhu L, Yi T. Dioscorea nipponica Makino: a systematic review on tis ethnobotany, phytochemical and pharmacological profiles. Pharmacogn Mag. 2018;11(43):567-73.

18. Zhou Q, Lin FF, Liu SM, Sui XF. Influence of the total saponin fraction from Dioscorea.nipponica Makino on TLR2/4-IL-1R receptor signal pathway in rats of gouty arthritis. J Ethnopharmacol. 2017;206:274-82.

19. Zhou Q, Yu DH, Zhang N, Liu SM. Anti-inflammatory effect of total saponin fraction from Diosscorea nipponica Makino on gouty arthritis and its influence on NALP3 inflammasome. Chin J Integr Med. 2019;25(9):663-70.

20. Zhou Q, Yu DH, Zhang N, Wang XY, Zhang N, Lin FF, et al. Therapeutic effects of total saponins from Dioscorea nippoonica Makino on gouty arthritis based on the MAPK-PPARy signaling pathway: an in vitro study. Nat Prod Commun. 2020;15(3):1-13.

21. Lu F, Liu L, Yu DH, Li XZ, Zhou Q, Liu SM, et al. Therapeutic effect of Rhizoma Dioscorea Nipponicae on gouty arthritis based on the SDF-1/ CXCR4 and p38 MAPK pathway: an in vivo and in vitro study. Phytother Res. 2013;28(2):280-8

22. Zhou Q, Yu DH, Zhang C, Lu F. Total saponins from Discorea nipponica ameliorate urate excretion. in hyperuricemia mice. Planta Med. 2014;80(15): 1259-68.

23. Cao XD, Wei RX, Zhou J, et al. Wenshen Jianpi recipe, a blended traditional Chinese medicine, ameliorates proteinuria and renal injury in a rat model of diabetic nephropathy. BMC Complement Altern Med. 2019;19:193.

24. Zhou F, Mei JT, Han XG, Li HJ, Yang SB, Wang MQ, et al. Kinsenoside attenuates osteoarthritis by repolarizing macrophages through inactivating NF-KB/MAPK signaling and protecting chondrocytes. Acta Pharm Sin B. 2019;9(5):973-85.

25. Zhang H, Huang XD, Shi Y, Liu WG, He MX. Identification and analysis of an MKK4 homologue in response to the nucleus grafting operation and antigens in the pearl oysterpinctada fucata. Fish Shellfish Immunol. 2018;73:279-87.

26. Wang HB, Huang SH, Xu M, Yang J, Yang J, Liu MX, et al. Galangin ameliorates cardiac remodeling via the MEK1/2-ERK1/2 and PI3K-AKT pathway. J Cell Physiol. 2019;234(9):15654-67.

27. Stramucci L, Pranteda A, Bossi G. Insights of crosstalk between p53 protein and the MKK3/MKK6/P38 MAPK signaling pathway in cancer. Cancers (Basel). 2018;10(5):131

28. Wang RC, Jiang DM. PPARy agonist pioglitazone affects rat gouty arthritis by regulating cytokines. Genet Mol Res. 2014;13(3):6577-81.

29. Luo SF, Chin CY, Ho L, Tseng WY, Kuo CF, Lai JH. Monosodium urate crystals induced ICAM-1 expression and cell-cell adhesion in renal mesangial cells: implications for the pathogenesis of gouty nephropathy. J Microbiol Immunol Infect. 2020;53(1):23-32.

30. Li X, Meng XR, He YZ, Spiliopoulou A, Timofeeva M, Wei WQ, et al. Genetically determined serum urate levels and cardiovascular and other diseases in UK biobank cohort: a phenome-wide mendelian randomization study. PLoS Med. 2019;16(10):e1002937.

\section{Publisher's Note}

Springer Nature remains neutral with regard to jurisdictional claims in published maps and institutional affiliations.

Ready to submit your research? Choose BMC and benefit from:

- fast, convenient online submission

- thorough peer review by experienced researchers in your field

- rapid publication on acceptance

- support for research data, including large and complex data types

- gold Open Access which fosters wider collaboration and increased citations

- maximum visibility for your research: over $100 \mathrm{M}$ website views per year

At BMC, research is always in progress.

Learn more biomedcentral.com/submission 